\title{
Are we missing the target? Are we aiming too low? What are the aerobic exercise prescriptions and their effects on markers of cardiovascular health and systemic inflammation in patients with knee osteoarthritis? A systematic review and meta-analysis
}

\author{
Jenna M Schulz, ${ }^{1,2}$ Trevor B Birmingham (D) , 1,2 Hayden F Atkinson, ${ }^{1,2}$ Emilie Woehrle, ${ }^{3}$ \\ Codie A Primeau, 1,2 Michael J Lukacs, 1,2 Baraa K Al-Khazraji, ${ }^{2,3}$ Michaela C M Khan, 2,3 \\ Bryn 0 Zomar, ${ }^{1,2}$ Robert J Petrella, ${ }^{2,4}$ Frank Beier, ${ }^{2,5}$ C Thomas Appleton, 2,6 \\ J Kevin Shoemaker, ${ }^{2,3}$ Dianne M Bryant ${ }^{1,2}$
}

- Additional material is published online only. To view please visit the journal online (http://dx.doi.org/10.1136/ bjsports-2018-100231).

${ }^{1}$ Health and Rehabilitation Sciences, Western University Faculty of Health Sciences, London, Ontario, Canada ${ }^{2}$ Bone and Joint Institute, Western University, London, Ontario, Canada

${ }^{3}$ Kinesiology, Western University Faculty of Health Sciences, London, Ontario, Canada ${ }^{4}$ Family Medicine, Western University Schulich School of Medicine and Dentistry, London, Ontario, Canada

${ }^{5}$ Physiology and Pharmacology, Western University Schulich School of Medicine and Dentistry, London, Ontario, Canada

${ }^{6}$ Medicine, Western University Schulich School of Medicine and Dentistry, London, Ontario, Canada

Correspondence to Dr Trevor B Birmingham, Faculty of Health Sciences, Bone and Joint Institute, London, ON N6G 1H1, Canada; tbirming@uwo.ca

Accepted 17 November 2019 Published Online First 17 December 2019

Check for updates

(C) Author(s) (or their employer(s)) 2020. No commercial re-use. See rights and permissions. Published by BMJ.

To cite: Schulz JM, Birmingham TB, Atkinson HF, et al. Br I Sports Med

2020:54:771-775

\section{ABSTRACT}

Objectives We systemically reviewed published studies that evaluated aerobic exercise interventions in patients with knee osteoarthritis (OA) to: (1) report the frequency, intensity, type and time (FITT) of exercise prescriptions and (2) quantify the changes in markers of cardiovascular health and systemic inflammation.

Data sources PubMed, CINAHL, Scopus; inception to January 2019.

Eligibility criteria Randomised clinical trials (RCT), cohort studies, case series.

Design We summarised exercise prescriptions for all studies and calculated effect sizes with $95 \% \mathrm{Cls}$ for between-group (RCTs that compared exercise and control groups) and within-group (pre-post exercise) differences in aerobic capacity $\left(\mathrm{VO}_{2}\right)$, heart rate $(\mathrm{HR})$, systolic blood pressure (SBP), diastolic blood pressure (DBP) and inflammatory markers (interleukin-6 (IL-6), tumour necrosis factor-alpha). We pooled results where possible using random effects models.

Results Interventions from 49 studies were summarised; 8\% (4/49) met all FITT guidelines; 16\% (8/49) met all or most FITT guidelines. Fourteen studies (10 RCTs) reported at least one marker of cardiovascular health or systemic inflammation. Mean differences (95\% $\mathrm{Cl})$ indicated a small to moderate increase in $\mathrm{VO}_{2}(0.84$ $\mathrm{mL} / \mathrm{min} / \mathrm{kg} ; 95 \% \mathrm{Cl} 0.37$ to 1.31$)$, decrease in $\mathrm{HR}(-3.56$ beats per minute; $95 \% \mathrm{Cl}-5.60$ to -1.52$)$ and DBP $(-4.10 \mathrm{~mm} \mathrm{Hg} ; 95 \% \mathrm{Cl}-4.82$ to -3.38$)$ and no change in SBP $(-0.36 \mathrm{~mm} \mathrm{Hg} ; 95 \% \mathrm{Cl}-3.88$ to 3.16$)$ and IL-6 (0.37 pg/mL; $95 \% \mathrm{Cl}-0.11$ to 0.85$)$. Within-group differences were also small to moderate.

Conclusions In studies of aerobic exercise in patients with knee $0 A$, very few interventions met guidelinerecommended dose; there were small to moderate changes in markers of cardiovascular health and no decrease in markers of systemic inflammation. These findings question whether aerobic exercise is being used to its full potential in patients with knee OA. PROSPERO registration number CRD42018087859.

\section{INTRODUCTION}

The burden of knee osteoarthritis (OA) is substantial and growing. ${ }^{1}$ Patients with knee OA have multiple comorbidities including obesity, cardiovascular disease, diabetes and metabolic syndrome. ${ }^{1-4}$ Knee $\mathrm{OA}$ is associated with cardiovascular events and all-cause mortality. ${ }^{56}$ Mechanisms of OA progression include chronic low-grade inflammation, vascular endothelial dysfunction and metabolic disturbances. ${ }^{27-9}$ Markers of systemic inflammation are associated with increased knee OA structural progression and increased pain. ${ }^{10-12}$

Given the well-established effects of aerobic exercise on cardiovascular health and systemic inflammation, ${ }^{13} 14$ there is strong rationale for its therapeutic use in patients with knee OA. Aerobic exercise is recommended for chronic diseases because of its ability to improve multiple physiological impairments, ${ }^{13} 1516$ and does so in a doserelated manner; higher frequency and intensity of exercise improves cardiovascular health, ${ }^{17} 18$ expends calories ${ }^{19}$ and reduces chronic inflammation. ${ }^{2021}$ The American College of Sports Medicine (ACSM) provides guidelines for aerobic exercise prescription expressed as the frequency, intensity, type and time (FITT) principle (online supplementary table 1). ${ }^{22}$

Exercise programmes of various types (ie, aerobic, strength, neuromuscular) reduce pain and increase function immediately after treatment in patients with knee OA, ${ }^{2324}$ but the effects of aerobic exercise beyond musculoskeletal outcomes are unclear. $^{23} 25$ Despite strong physiological rationale for aerobic interventions, the exercise prescriptions and changes in markers of cardiovascular health and systemic inflammation have not been systematically reviewed in patients with knee OA.

Therefore, we systemically reviewed published studies in patients with knee OA that evaluated the effect of aerobic exercise interventions to: (1) report the exercise prescriptions according to the FITT principle and (2) quantify the changes in markers of cardiovascular health and systemic inflammation.

\section{METHODS}

\section{Protocol and registration}

This study conformed to Preferred Reporting Items for Systematic Reviews and Meta-Analyses 
methodological guidelines ${ }^{26}$ and the Cochrane Handbook ${ }^{27}$ and has been registered in PROSPERO.

\section{Eligibility criteria}

We included randomised clinical trials (RCT), cohort studies and case series that evaluated an aerobic exercise intervention (strictly aerobic or mixed interventions that included an aerobic portion) in patients diagnosed with knee OA (tibiofemoral and/ or patellofemoral; unilateral and/or bilateral). The interventions were considered aerobic as defined by ACSM; a mode of physical activity that results in improvement and maintenance of cardiorespiratory function, including walking, running, swimming, cycling and dancing. ${ }^{22}$ Studies must have compared outcome measures to a control group (between-group) or prepost aerobic exercise (within-group).

\section{Search}

We searched PubMed, CINAHL and Scopus from database inception to January 2019. The search terms included [Osteoarthritis AND (knee OR knees OR knee joint OR knee joints) AND (exercise OR aerobic OR walk OR walking OR run OR running OR cycle OR cycling OR jog OR jogging OR swim OR swimming OR dance OR dancing)]. Additionally, we manually searched reference lists from previous systematic reviews. Studies were restricted to those written in either English or French. Eight reviewers (four pairs) independently examined one-quarter of the titles and abstracts and classified each to exclude or retrieve for full-text review, using Covidence (Covidence systematic review software, Veritas Health Innovation, Melbourne, Australia, www.covidence.org). The same eight reviewers independently assessed full-text articles for eligibility. One reviewer (JMS) instructed all other reviewers on study eligibility (titles, abstracts and full-text review) and data extraction procedures. If the decision regarding eligibility differed among any pair, the same reviewer (JMS) consulted with the pair to reach consensus.

\section{Data extraction}

Each reviewer individually extracted data for: study design, age, sex, body mass index (BMI), OA diagnosis (ie, radiographic and Kellgren and Lawrence grade if provided and/or symptomatic OA) and characteristics of the exercise programme including type of exercise, frequency (sessions/week), intensity (heart rate $\left(\% \mathrm{HR}_{\max }\right) /$ aerobic capacity $\left(\% \mathrm{VO}_{2 \max }\right) /$ heart rate reserve $(\% \mathrm{HRR}) /$ rating of perceived exertion), time (minutes/session, including warm-up and cool down), duration of intervention (number of weeks), presence of a supervisor and the extent to which ACSM criteria were met.

For studies that reported a marker of cardiovascular health or systemic inflammation, we extracted the method used to assess the outcome and associated statistics for preintervention and postintervention scores in all groups (and subgroups) involved. For all studies, unadjusted mean differences (MD) were extracted, unless reported otherwise (two $\mathrm{RCTs}^{28}{ }^{29}$ reported adjusted means). Reported markers of cardiovascular health included $\mathrm{VO}_{2}(\mathrm{~mL} / \mathrm{min} / \mathrm{mm} \mathrm{Hg})$, HR (beats per minute; bpm), systolic blood pressure (SBP; mm Hg) and diastolic blood pressure (DBP; $\mathrm{mm} \mathrm{Hg})$. Reported markers of systemic inflammation included interleukin-6 (IL-6; pg/mL) and tumour necrosis factoralpha (TNF- $\alpha ; \mathrm{pg} / \mathrm{mL}$ ). Additionally, as the $6 \mathrm{~min}$ walk test (6MWT (metres)) is a measure of cardiorespiratory fitness and musculoskeletal health, we evaluated it separately and included it in online supplementary appendix 1. Study authors were contacted if additional data were required, and manual estimates from figures were obtained if insufficient data were provided.

\section{Risk of bias}

We used the Cochrane Risk of Bias tool and the Risk of Bias in Non-randomized Studies of Interventions to rate the quality of RCTs and non-RCTs, respectively. ${ }^{30} 31$ The reviewers independently assessed each study using the same procedures described above, so that risk of bias for each study was rated by two reviewers and the same third reviewer (JMS) consulted to reach consensus for any discrepancies. Quality of the studies was ranked as 'low', 'some' and 'high' bias based on the following study design factors: randomisation, deviations from intended interventions, missing outcome data, measurement of outcome and selection of the reported result. Additionally, bias due to confounding, in the selection of participants and in the classification of the intervention, was rated for non-RCTs. An overall risk rating for the study was estimated from the individual risks of each domain. A weighted kappa $\left(\kappa_{\mathrm{w}}\right)$ for inter-rater agreement was calculated for each pair of reviewers before discrepancies were resolved (SPSS V.23, IBM). We also used the Grading of Recommendations Assessment, Development and Evaluation (GRADE) to rate the overall quality of evidence in the systematic reviews, ${ }^{32}$ and the Template for Intervention Description and Replication to aid in the description of the exercise interventions. ${ }^{33}$

\section{Synthesis of results}

We calculated the raw MD and 95\% CIs for each study and pooled data using random effects meta-analyses. We first analysed between-group differences (ie, aerobic exercise intervention vs control) from RCTs. We then analysed within-group (ie, prepost aerobic exercise) paired differences for all study designs. All but one study ${ }^{28}$ provided preintervention and postintervention or change data.

When not reported, the SD was estimated from the SE of the mean, 95\% CI, p value or other methods suggested in the Cochrane Handbook. ${ }^{27}$ Means and SDs were estimated from median and range for two studies. ${ }^{34}$ Following Cochrane guidelines, for any study that included two different intervention groups (ie, cycling vs walking) and one control group, the sample size in the control group was evenly divided so a comparison could be made to each intervention. ${ }^{27}$ We imputed $r=0.5$ when the correlation of prescores and postscores was required and we performed sensitivity analyses using $\mathrm{r}$ values ranging from 0.1 to $0.9 .^{35}{ }^{36}$ Heterogeneity was examined using the $\mathrm{I}^{2}$ statistic and $\mathrm{p}$ values, where an absence of inconsistency was represented as $0 \%$, less than $40 \%$ was considered low, 30\%-60\% was moderate, 50\%-90\% was substantial, 75\%-100\% was considerable and 100\% indicated maximal inconsistency (ie, high heterogeneity). ${ }^{37}$ Additional subgroup and sensitivity analyses were planned based on a priori hypotheses described in online supplementary appendix 1 . Meta-analyses were performed using Comprehensive Meta-Analysis software program (V.3, Biostat).

\section{RESULTS}

\section{Study selection}

Our electronic search yielded 5857 studies with 244 eligible for full-text review. Forty-nine studies met our eligibility criteria for objective 1 , review of exercise prescriptions (figure 1). ${ }^{28} 2938-83$ Twenty-three RCTs compared an aerobic exercise group to a nonexercising control group, 15 RCTs compared groups completing different types of exercise and 11 studies compared outcomes 


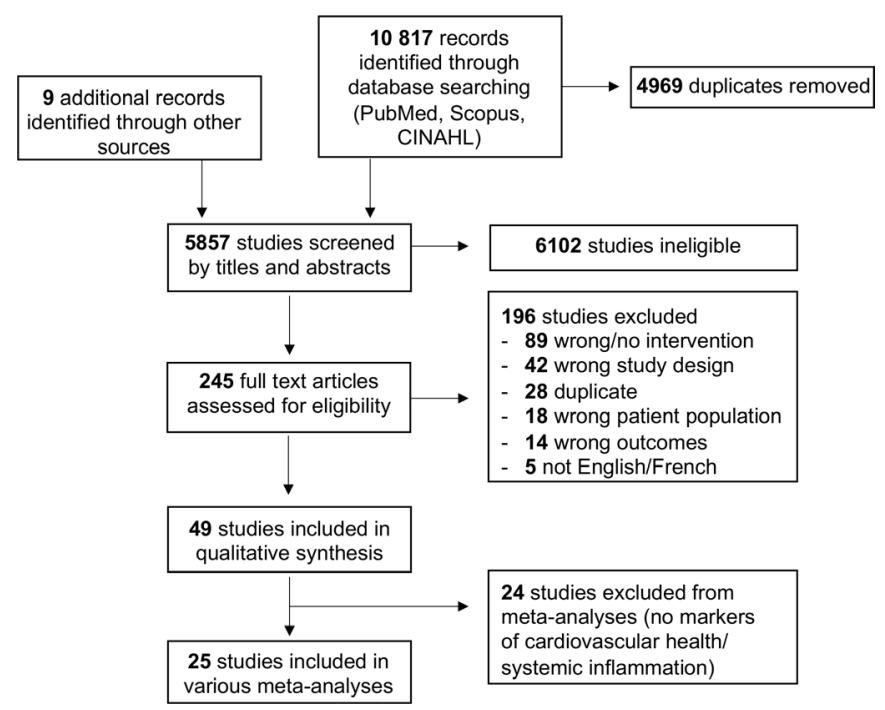

Figure 1 Preferred Reporting Items for Systematic Reviews and MetaAnalyses (PRISMA) flow chart of included and excluded studies.

before and after aerobic exercise in one group. Fourteen studies reported a marker of cardiovascular health and/or systemic inflammation and were included in various meta-analyses. For between-group analyses, 10 RCTs that included a control group were included (online supplementary table 2). Four studies compared $\mathrm{VO}_{2}$, three compared $\mathrm{HR}$, two compared SBP and DBP and three compared IL-6. For within-group analyses, all study types were included. In total, five studies reported $\mathrm{VO}_{2}$, four reported HR, three reported SBP and DBP, five reported IL- 6 and four reported TNF- $\alpha$ (online supplementary table 3).

\section{Participant and intervention characteristics}

A total of 3448 patients were included for objective 1 (76\% female, age $=63 \pm 7$ years; $\mathrm{BMI}=29.3 \pm 4.9 \mathrm{~kg} / \mathrm{m}^{2}$; mean $\left.\pm \mathrm{SD}\right)$. Three studies 3940 where patients had OA at multiple joints were included because over $60 \%$ of the participants had knee OA (online supplementary table 2).

\section{Comparison to ACSM guidelines for aerobic exercise}

Interventions in 2 of the 49 studies (4\%) met all domains of the ACSM FITT criteria, and another two ${ }^{46} 64$ met the criteria progressively over time, whereby the duration (ie, minutes/ session) and/or intensity (ie, $\% \mathrm{HR}_{\max } / \mathrm{VO}_{2 \max }$ ) increased as the intervention advanced forward. Therefore, all domains were met by the end of the intervention (4/49, 8\%). Interventions in another four studies $(8 \%)^{29} 697072$ met three of the FITT criteria recommended by ACSM, with the exception of 150 min of aerobic exercise/week. Therefore, 8/49 (16\%) met all or most of the criteria. All exercise interventions were classified as moderate intensity (or less) according to ACSM criteria (online supplementary table 1 ). The overall average patient adherence to the intervention was $86 \%$, measured by attendance or dropout (online supplementary table 3 ).

\section{Risk of bias}

There was substantial agreement on items with the exception of bias in the selection of participants and bias in the reporting of results for non-RCTs, requiring the third reviewer to reach consensus $\left(\mathrm{p}<0.05, \kappa_{\mathrm{w}}\right.$ ranging from 0.13 to 0.75$)$. For RCTs, $45 \%$ (17/38) of studies had 'high' risk for measurement bias. Most studies had 'low' or 'some' risk for selection, performance and detection bias. All non-RCTs had a high risk of bias for confounding, and most $(8 / 11,72 \%)$ had a high risk for intervention classification. Patient and personnel blinding was not possible in all studies; however, most had inappropriate methods to control for confounding variables or did not blind outcome assessors. All studies had a 'low' risk for participant selection bias, because selection was not based on characteristics observed after the start of the study. Overall, most studies (36/49, 74\%) had a 'high' risk of bias, 9 (18\%) had a 'low' risk of bias and $4(8 \%)$ had 'some' risk of bias (online supplementary table 3, online supplementary figure 1). Additionally, all studies rated low in the quality of evidence according to GRADE.

Markers of cardiovascular health and systemic inflammation Synthesis of results comparing aerobic exercise to control Between-group (RCT) analyses (MD, 95\% CI) indicated a statistically significant increase in $\mathrm{VO}_{2}(0.84 \mathrm{~mL} / \mathrm{min} / \mathrm{kg} ; 95 \%$ CI 0.37 to 1.3$)$, decrease in $\mathrm{HR}(-3.56 \mathrm{bpm}$; $95 \% \mathrm{CI}-5.60$ to -1.52$)$ and decrease in DBP $(-4.10 \mathrm{~mm} \mathrm{Hg} ; 95 \% \mathrm{CI}-4.82$ to -3.38$)$, $\mathrm{p}<0.05$ (figure $2 \mathrm{~A}, \mathrm{~B}, \mathrm{D})$. There were no statistically significant differences in SBP $(-0.36 \mathrm{~mm} \mathrm{Hg}$; 95\% CI -3.88 to 3.16$)$ and IL-6 (0.37 pg/mL; 95\% CI -0.11 to 0.85$)$; p > 0.05 (figure $2 \mathrm{C}, \mathrm{E}$ ).

Synthesis of results comparing pre-aerobic to post-aerobic exercise The within-group (pre-post exercise) analyses indicated a statistically significant increase in $\mathrm{VO}_{2}(1.69 \mathrm{~mL} / \mathrm{min} / \mathrm{kg} ; 95 \% \mathrm{CI}$ 0.51 to 2.87$)$, decrease in HR ( $-4.54 \mathrm{bpm}$; $95 \% \mathrm{CI}-6.82$ to $-2.25)$ and decrease in SBP $(-7.19 \mathrm{~mm} \mathrm{Hg}$; 95\% CI -11.40 to -2.25$)$ and DBP $(-4.66 \mathrm{~mm} \mathrm{Hg} ; 95 \% \mathrm{CI}-6.03$ to -3.28$)$ (online supplementary appendix 1 ; figure 2A-D). There was no statistically significant change in IL-6 $(-0.09 \mathrm{pg} / \mathrm{mL} ; 95 \%$ $\mathrm{CI}-0.63$ to $0.44, \mathrm{p}>0.05$ ) (online supplementary appendix 1 ; figure $2 \mathrm{E})$ and a statistically significant increase in TNF- $\alpha(0.99$ $\mathrm{pg} / \mathrm{mL}$; 95\% CI 0.21 to $1.77, \mathrm{p}<0.05$ ) (online supplementary appendix 1; figure $2 \mathrm{~F}$ ).

Results for additional analyses including 6MWT are reported in online supplementary appendix 1 (figure 3). Results of subgroup and sensitivity analyses were generally consistent with the primary analysis(online supplementary appendix 1; table 4).

\section{DISCUSSION}

What are the aerobic exercise prescriptions studied in patients with knee $0 A$ ?

Only $16 \%(8 / 49)$ of published studies in patients with knee OA evaluated interventions that met all or most of the ACSM guidelines for aerobic exercise prescription. Most interventions reviewed $(45 / 49,92 \%)$ did not meet the target of 150 $\mathrm{min} /$ week of moderate to vigorous activity. Additionally, most studies $(40 / 49,82 \%)$ did not measure $\mathrm{HR}$ or $\mathrm{VO}_{2}$ to ensure patients were exercising at the target intensity. Several exercise programmes $(22 / 49,45 \%)$ were less than 12 weeks; durations ranged from 4 to 78 weeks (online supplementary table 2). Most studies had a 'high' risk of bias (online supplementary table 3, online supplementary figure 1); and all studies rated low in the quality of evidence according to GRADE.

Why are the interventions not meeting recommended dose? We suggest the likely reasons the studied interventions did not strictly follow aerobic exercise guidelines include the perceived lack of ability for patients with knee OA to adhere to the guidelines, the fear of exacerbating symptoms and increasing adverse events and/or the desire to limit patients from dropping out. While well accepted as providing the physiological dose required to evoke 


$\begin{array}{lccccccc}\text { A) Study (subgroup) } & \begin{array}{c}\text { MD } \\ (\mathbf{m L} / \mathbf{m i n} / \mathbf{K g})\end{array} & \begin{array}{c}\text { Lower } \\ \text { limit }\end{array} & \begin{array}{c}\text { Upper } \\ \text { limit }\end{array} & \begin{array}{r}\text { Z-Value } \\ \text { p-Value }\end{array} & \text { Exercise } & \text { Control } \\ \text { 1997 Etinger } & 0.80 & 0.25 & 1.35 & 2.83 & <0.001 & 117 & 127 \\ \text { 2004 Keefe } & 2.90 & -1.57 & 7.37 & 1.27 & 0.20 & 16 & 18 \\ \text { 2013 Larose (female) } & -0.40 & -3.36 & 2.56 & -0.27 & 0.79 & 30 & 11 \\ \text { 2013 Larose (male) } & -1.70 & -6.77 & 3.37 & -0.66 & 0.51 & 12 & 21 \\ \text { 2015 Koli } & 1.10 & 0.14 & 2.06 & 2.24 & 0.03 & 36 & 40 \\ \text { Overall } & 0.84 & 0.37 & 1.31 & 3.52 & <0.001 & 211 & 217\end{array}$

Test for Heterogeneity: $I^{2}=0 \%, p=0.60$
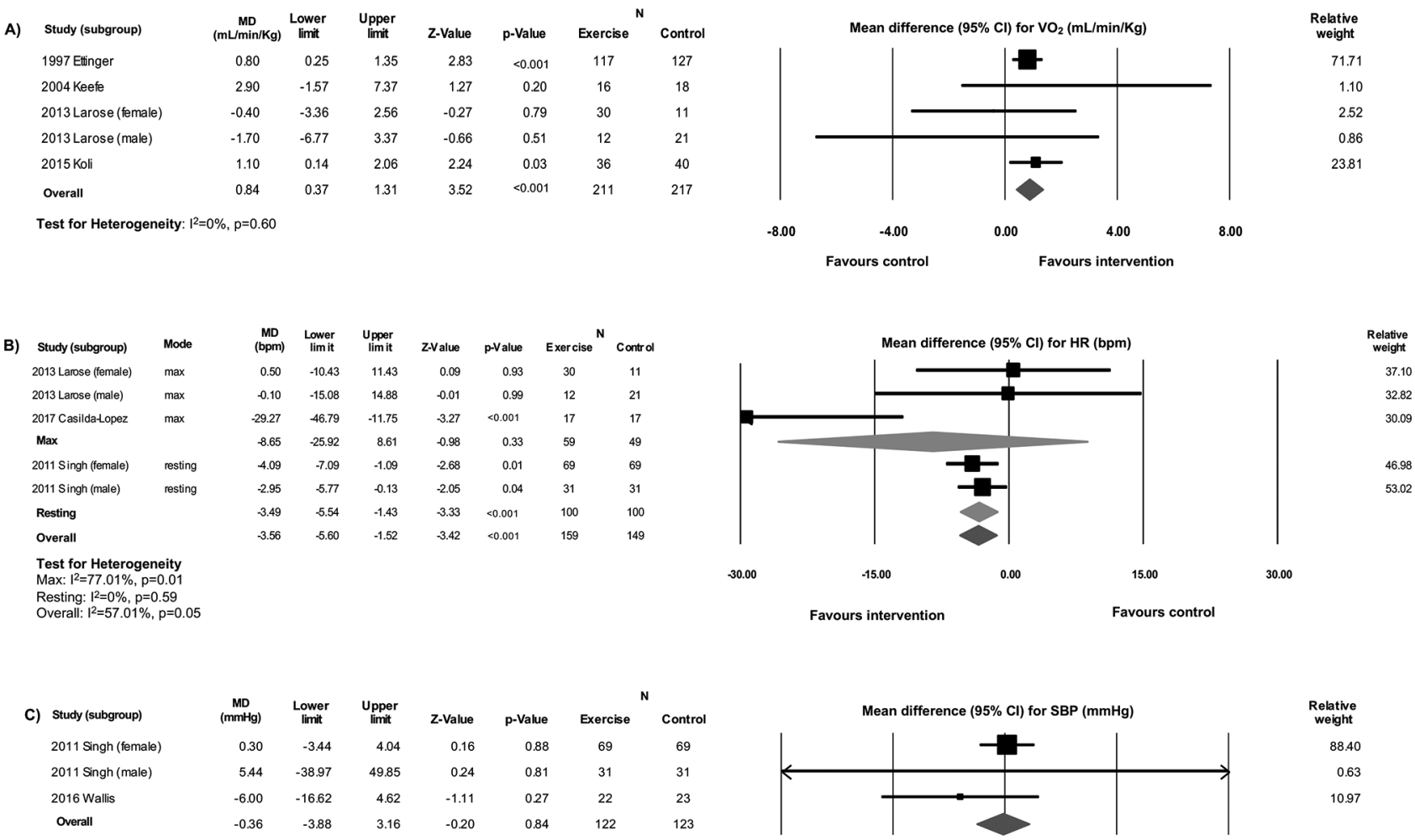

Test for Heterogeneity: $12=0 \%, p=0.53$

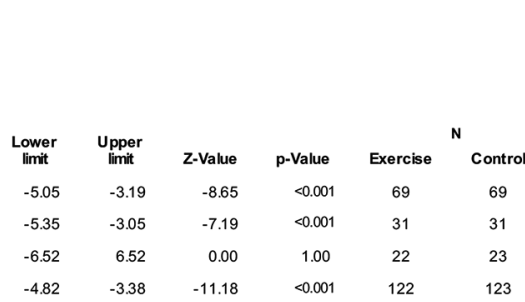

Test for Heterogeneity: $\left.\right|^{2}=0 \%, p=0.4$

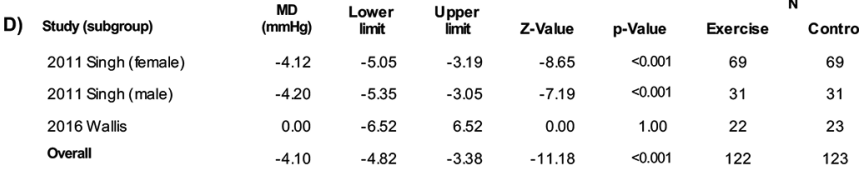

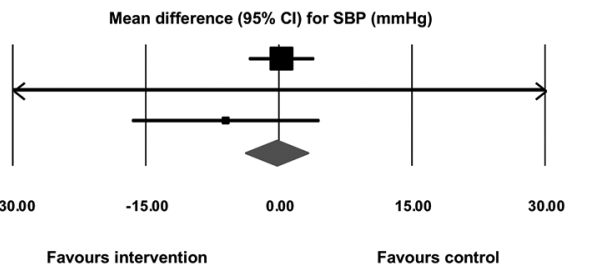

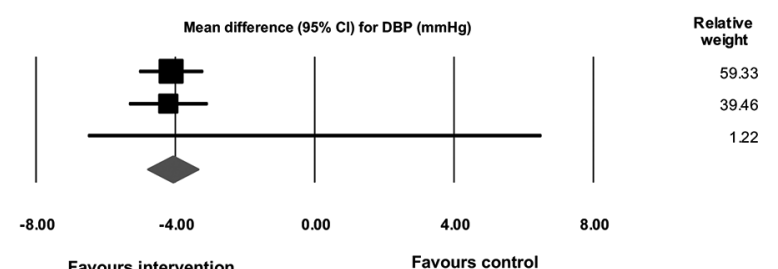

$\begin{array}{lrrrrrrr}\text { E) Study (subgroup) } & \begin{array}{c}\mathrm{MD} \\ (\mathrm{pg} / \mathrm{mL})\end{array} & \begin{array}{c}\text { Lower } \\ \text { limit }\end{array} & \begin{array}{c}\text { Upper } \\ \text { limit }\end{array} & \text { Z-Value } & \mathrm{p} \text {-Value } & \text { Exercise } & \text { Control } \\ \text { 2004 Nicklas } & 0.25 & -0.72 & 1.22 & 0.51 & 0.61 & 53 & 60 \\ 2014 \text { Messier } & 0.60 & 0.09 & 1.11 & 2.29 & 0.02 & 134 & 129 \\ 2015 \text { Samut } & -0.36 & -1.52 & 0.80 & -0.60 & 0.55 & 14 & 13 \\ \text { Overall } & 0.37 & -0.11 & 0.85 & 1.50 & 0.13 & 201 & 202\end{array}$

Test for Heterogeneity: $\left.\right|^{2}=13.34 \%, p=0.31$

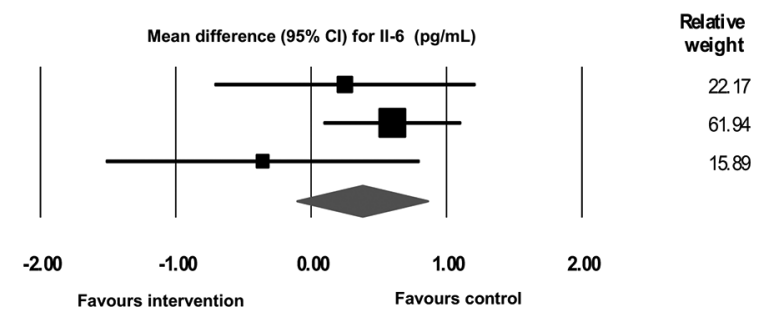

Figure 2 Forest plot of between-group analyses (ie, randomised clinical trials (RCT); exercise vs control) for the following outcomes: aerobic capacity $\left(\mathrm{VO}_{2}, \mathrm{~A}\right)$; heart rate $(\mathrm{HR}, \mathrm{B})$; systolic blood pressure (SBP, C); diastolic blood pressure (DBP, D); and interleukin-6 (IL-6, E). Weights are from a random effects analysis. Overall effects are shown as mean difference (MD) with $95 \% \mathrm{Cl}$. N, number of patients.

improvements in cardiovascular health and chronic inflammation, the FITT guidelines are not disease specific. Therefore, patients and their health providers must adjust guideline parameters to meet individual patient needs. ${ }^{84}$ Other recently published meta-analyses suggest that patients with knee OA do not experience increased pain with exercise, ${ }^{24}$ and that knee loading with exercise is not harmful to the articular cartilage. ${ }^{85}$ Therefore, published exercise prescription guidelines are recommended to guide exercise interventions for people with OA. ${ }^{86}$

Aerobic exercise in patients for other chronic diseases has been able to meet ACSM guidelines. In patients with type 2 diabetes, higher intensities of aerobic exercise resulted in greater improvements in cardiorespiratory fitness. ${ }^{87} \mathrm{~A}$ recent systematic review of the effects of aerobic exercise for patients with metabolic syndrome only included studies with interventions that met the FITT criteria. It reported substantial improvements in HR, SBP and $\mathrm{VO}_{2}$, with vigorous intensity interventions providing greater effects. ${ }^{88}$

What are the effects on markers of cardiovascular health and systemic inflammation?

Only 14 of the studies (29\%) evaluated the effects of aerobic exercise using a marker of cardiovascular health or systemic 
inflammation. Meta-analyses of those results indicated only small to moderate improvements in $\mathrm{VO}_{2}, \mathrm{HR}$ and DBP (online supplementary appendix 1; figure 2). Although some of these improvements may arguably be clinically important, they are less than typically observed following aerobic exercise interventions and are likely to fall short in evoking optimal effects for patients with knee OA. As administered in the studies reviewed, the aerobic exercise interventions did not decrease circulating inflammatory factors IL- 6 and TNF- $\alpha$ (online supplementary appendix 1; figure 2).

When ACSM guideline-recommended dose is met, aerobic exercise can markedly improve cardiovascular health, ${ }^{89} 90$ decrease systemic inflammation ${ }^{9192}$ and increase caloric expenditure. ${ }^{19}$ Such physiological outcomes have potential to affect multiple disease processes linked to $\mathrm{OA}^{93-95}$ and may be reasonably hypothesised to decrease the risk of progression of knee $\mathrm{OA}$ and associated comorbidities. The caveat lies in the proper administration and adherence to aerobic exercise guidelines. Importantly, as suggested by the present data, when patients with OA are not meeting recommended guidelines, optimal improvements in cardiovascular health and systemic inflammation do not occur. Without such physiological changes, there may be no concomitant improvement in OA progression or comorbidities, and improvements in pain and function may be less than possible. Therefore, the true potential of aerobic exercise as a treatment for knee OA may be unrealised.

It is important to acknowledge that despite not meeting ACSM guidelines, the interventions were generally well attended by patients and did provide small to moderate improvements in some markers of cardiovascular health (online supplementary appendix 1; figure 2). As studied, the interventions also provided moderate improvements in the 6MWT (online supplementary appendix 1; figure 3).The results of the present review do not negate the importance of encouraging patients with knee OA to exercise, even at lower levels than those suggested to evoke optimal changes in cardiovascular health and systemic inflammation, given the other health benefits of physical activity. ${ }^{24} 96-99$

\section{Limitations}

The present estimates of the within-group pre-post exercise changes (online supplementary appendix 1 ; figure 2 ) should be interpreted carefully. For these analyses, the true intervention effects may not be discernible from effects caused by the simple passage of time, measurement error and/or characteristics of the patients and setting. ${ }^{100}$ Similarly, including non-randomised studies may contribute to uncontrolled confounding. Regardless, our between-group and within-group comparisons reveal lower changes in cardiovascular health and systemic inflammation than previously observed in randomised trials of aerobic exercise in patients with other chronic diseases. ${ }^{8788}$

The present methods used to determine effect sizes assume normally distributed outcomes within each study and this may not always be the case, especially for the several relatively small studies included in this review. Moreover, although studies reported the same markers, pooled data across all studies were sometimes assessed using slightly different methods (ie, calculated max vs peak $\mathrm{VO}_{2}$, using a treadmill protocol vs cycling protocol, and so on). While we reported individual and pooled study effect sizes, and performed sensitivity analyses where possible, results may differ based on study methods. Lastly, few studies reported sex-specific analyses. These should be performed in future, adequately powered studies.

\section{CONCLUSIONS}

Despite the strong rationale to improve cardiovascular health and reduce systemic inflammation in patients with knee OA, published studies include aerobic exercise interventions that rarely meet recommended dose guidelines. The pooled results from studies reporting a marker of cardiovascular health or systemic inflammation suggest only small to moderate effects on $\mathrm{VO}_{2}, \mathrm{HR}$ and blood pressure and no decrease in inflammatory markers. Therefore, clinicians may not be extracting the full potential benefit of aerobic exercise in patients with knee OA. Whether this is the case or not needs to be studied.

\section{What is already known}

Knee osteoarthritis $(\mathrm{OA})$ is associated with systemic inflammation, cardiovascular events and all-cause mortality.

- Aerobic exercise improves cardiovascular health and reduces systemic inflammation - this is a strong rationale for its use in patients with knee $\mathrm{OA}$.

\section{What are the new findings}

- In studies of aerobic exercise in patients with knee $\mathrm{OA}$, the interventions did not meet recommended exercise dose; postexercise anticipated increase in $\mathrm{VO}_{2}$ and decreases in heart rate and blood pressure were small to moderate; there was no decrease in systemic inflammatory markers interleukin-6 and tumour necrosis factor-alpha.

- The therapeutic effects of aerobic exercise in patients with knee OA remain unclear. We encourage studies of interventions that evoke more substantial changes in cardiovascular health and systemic inflammation.

Twitter Jenna M Schulz @jennaschulz_1

Contributors JMS conceptualised the project, analysed the data, and drafted and edited the manuscript. TBB, RJP, FB and DMB contributed to conception and design and critical revision of the article. DMB provided statistical expertise. HFA, EW, CAP, MJL, BKA, MCMK and BOZ contributed to data collection and synthesis. CTA and JKS provided critical analysis of the data and critical revision of the article. All authors edited and approved the final version of the manuscript.

Funding JMS is funded by a Transdisciplinary Training Award from the Collaborative Training Specialization in Musculoskeletal Health Research, Bone and Joint Institute, Western University. TBB, JKS and FB are funded in part by the Canada Research Chairs Program. Support for this study was provided by the Canadian Institutes of Health Research and The Arthritis Society, Canada.

Competing interests None declared.

Patient consent for publication Not required.

Provenance and peer review Not commissioned; externally peer reviewed.

ORCID iD

Trevor B Birmingham http://orcid.org/0000-0002-3300-7677

\section{REFERENCES}

1 James SL, Abate D, Abate KH, et al. Global, regional, and national incidence, prevalence, and years lived with disability for 354 diseases and injuries for 195 countries and territories, 1990-2017: a systematic analysis for the global burden of disease study 2017. The Lancet 2018:392:1789-858.

2 Roos EM, Arden NK. Strategies for the prevention of knee osteoarthritis. Nat Rev Rheumatol 2016:12:92-101.

3 McAlindon TE, Bannuru RR, Sullivan MC, et al. OARSI guidelines for the non-surgical management of knee osteoarthritis. Osteoarthritis Cartilage 2014;22:363-88

4 Felson DTet al. Osteoarthritis: new insights. Part 1: the disease and its risk factors. Ann Intern Med 2000;133:635-9.

5 Hawker GA, Croxford R, Bierman AS, et al. All-Cause mortality and serious cardiovascular events in people with hip and knee osteoarthritis: a population based cohort study. PLoS One 2014;9:e91286.10.1371/journal.pone.0091286 
6 Nüesch E, Dieppe P, Reichenbach S, et al. All cause and disease specific mortality in patients with knee or hip osteoarthritis: population based cohort study. BMJ 2011;342.

7 Sowers M. Epidemiology of risk factors for osteoarthritis: systemic factors. Curr Opin Rheumatol 2001;13:447-51.

8 Loeser RF. aging and osteoarthritis. Curr Opin Rheumatol 2012;23:492-6.

9 Rahmati M, Mobasheri A, Mozafari M. Inflammatory mediators in osteoarthritis: a critical review of the state-of-the-art, current prospects, and future challenges. Bone 2016;85:81-90.

10 Greene MA, Loeser RF. Aging-Related inflammation in osteoarthritis. Osteoarthritis Cartilage 2015;23:1966-71.

11 Stannus OP, Jones G, Blizzard L, et al. Associations between serum levels of inflammatory markers and change in knee pain over 5 years in older adults: a prospective cohort study. Ann Rheum Dis 2013;72:535-40.

12 Livshits G, Zhai G, Hart DJ, et al. Interleukin-6 is a significant predictor of radiographic knee osteoarthritis: the Chingford study. Arthritis Rheum 2009:60:2037-45.

13 Myers J. Exercise and cardiovascular health. Circulation 2003;107:e2-5.

14 Zheng G, Qiu P, Xia R, et al. Effect of aerobic exercise on inflammatory markers in healthy middle-aged and older adults: a systematic review and meta-analysis of randomized controlled trials. Front Aging Neurosci 2019;11.

15 Woods JA, Wilund KR, Martin SA, et al. Exercise, inflammation and aging. Aging Dis 2012;3:130-40.

16 Martin M, Krystof S, jiri R, et al. Modulation of energy intake and expenditure due to habitual physical exercise. Curr Pharm Des 2016;22:3681-99.

17 Swain DP, Franklin BA. Comparison of cardioprotective benefits of vigorous versus moderate intensity aerobic exercise. Am J Cardiol 2006;97:141-7.

18 Sattelmair J, Pertman J, Ding EL, et al. Dose response between physical activity and risk of coronary heart disease. Circulation 2011;124:789-95.

19 Hunter GR, Weinsier RL, Bamman MM, et al. A role for high intensity exercise on energy balance and weight control. Int J Obes 1998;22:489-93.

20 Colbert LH, Visser M, Simonsick EM, et al. Physical activity, exercise, and inflammatory markers in older adults: findings from the health, aging and body composition study. J Am Geriatr Soc 2004; 52:1098-104.

21 Reuben DB, Judd-Hamilton L, Harris TB, et al. The associations between physical activity and inflammatory markers in high-functioning older persons: MacArthur studies of successful aging. J Am Geriatr Soc 2003;51:1125-30.

22 Reibe D, Jonathan K, Liguori G, et al. ACSM's Guidelines for Exercise Testing and Prescription. 10th edn. Wolters Kluter, 2018.

23 Verhagen AP, Ferreira M, Reijneveld-van de Vendel EAE, et al. Do we need another trial on exercise in patients with knee osteoarthritis?: no new trials on exercise in knee oa. Osteoarthr Cartil 2019;27:1266-9.

24 Juhl C, Christensen R, Roos EM, et al. Impact of exercise type and dose on pain and disability in knee osteoarthritis: a systematic review and meta-regression analysis of randomized controlled trials. Arthritis Rheumatol 2014;66:622-36.

25 Bricca $A$, Lund $H$, Roos EM, et al. When enough is enough - How to determine when the evidence for the effectiveness of a treatment is sufficient? Osteoarthritis and Cartilage 2019;27:1253-6.

26 Liberati A, Altman DG, Tetzlaff J, et al. The PRISMA statement for reporting systematic reviews and meta-analyses of studies that evaluate healthcare interventions: explanation and elaboration. BMJ 2009;339:b2700.

27 Higgins J, Green S. Cochrane Handbook for systematic reviews of interventions version 5.1.0, 2011. Available: http://handbook-5-1.cochrane.org/

28 Ettinger WHet al. A randomized trial comparing aerobic exercise and resistance exercise with a health education program in older adults with knee osteoarthritis. JAMA 1997;277:25-31.

29 Messier SP, Mihalko S, Loeser RF, et al. Glucosamine/chondroitin combined with exercise for the treatment of knee osteoarthritis: a preliminary study. Osteoarthritis and Cartilage 2007:15:1256-66.

30 Higgins JPT, Altman DG, Gøtzsche PC, et al. The Cochrane collaboration's tool for assessing risk of bias in randomised trials. BMJ 2011;343:d5928-9.

31 Sterne JAC, Hernán MA, Reeves BC, et al. ROBINS-I: a tool for assessing risk of bias in non-randomised studies of interventions. BMJ 2016;355:i4919-10.10.1136/bmj. i4919

32 Guyatt GH, Oxman AD, Vist GE, et al. Grade: an emerging consensus on rating quality of evidence and strength of recommendations. BMJ 2008;336:924-6.

33 Hoffmann TC, Glasziou PP, Boutron I, et al. Better reporting of interventions: template for intervention description and replication (TIDieR) checklist and guide. BMJ 2014;348:g1687-12.

34 Hozo SP, Djulbegovic B, Hozo I. Estimating the mean and variance from the median, range, and the size of a sample. BMC Med Res Methodol 2005;5:1-10.

35 Borenstein M. Effect sizes for continuous data. In: Cooper H, Hedges L, Valentine JC, eds. Handbook of research synthesis and meta-analysis. New York: Russel Sage Foundation, 2009: 221-36

36 Follmann D, Elliott $\mathrm{P}$, Suh I, et al. Variance imputation for overviews of clinical trials with continuous response. J Clin Epidemiol 1992;45:769-73

37 Higgins JPTet al. Measuring inconsistency in meta-analyses. BMJ 2003;327:557-60.
38 Ağlamiş B, Toraman NF, Yaman H. The effect of a 12-week supervised multicomponent exercise program on knee oa in Turkish women. J Back Musculoskelet Rehabil 2008;21:121-8.

39 Alkatan M, Baker JR, Machin DR, et al. Improved function and reduced pain after swimming and cycling training in patients with osteoarthritis. J Rheumatol 2016;43:666-72.

40 Alkatan M, Machin DR, Baker JR, et al. Effects of swimming and cycling exercise intervention on vascular function in patients with osteoarthritis. Am J Cardiol 2016;117:141-5

41 Bautch JC, Malone DG, Vailas AC, et al. Effects of exercise on knee joints with osteoarthritis: a pilot study of biologic markers. Arthritis Care Res 1997;10:48-55.

42 Beckwée D, Vaes P, Raeymaeckers S, et al. Exercise in knee osteoarthritis: do treatment outcomes relate to bone marrow lesions? A randomized trial. Disabil Rehabil 2017;39:1847-55.

43 Benli Küçük E, Ö Özyemişci Taşkıran, Tokgöz N, et al. Effects of isokinetic, isometric, and aerobic exercises on clinical variables and knee cartilage volume using magnetic resonance imaging in patients with osteoarthritis. Turkiye Fiz Tip ve Rehabil Derg 2018;64:8-16.

44 Bressel E, Wing JE, Miller Al, et al. High-Intensity interval training on an aquatic treadmill in adults with osteoarthritis. J Strength Cond Res 2014;28:2088-96.

45 Brooks MA, Beaulieu JE, Severson HH, et al. Web-Based therapeutic exercise resource center as a treatment for knee osteoarthritis: a prospective cohort pilot study. BMC Musculoskelet Disord 2014;15:1-11.

46 Brosseau L, Wells GA, Kenny GP, et al. The implementation of a community-based aerobic walking program for mild to moderate knee osteoarthritis: a knowledge translation randomized controlled trial: Part II: clinical outcomes. BMC Public Health 2012;12:1073.

47 Casilda-López J, Valenza MC, Cabrera-Martos I, et al. Effects of a dance-based aquatic exercise program in obese postmenopausal women with knee osteoarthritis: a randomized controlled trial. Menopause 2017;24:768-73.

48 Cheung C, Wyman JF, Bronas U, et al. Managing knee osteoarthritis with yoga or aerobic/strengthening exercise programs in older adults: a pilot randomized controlled trial. Rheumatol Int 2017:37:389-98.

49 de Rooij M, van der Leeden M, Cheung J, et al. Efficacy of tailored exercise therapy on physical functioning in patients with knee osteoarthritis and comorbidity: a randomized controlled trial. Arthritis Care Res 2017;69:807-16.

50 Evcik D, Sonel B. Effectiveness of a home-based exercise therapy and walking program on osteoarthritis of the knee. Rheumatol Int 2002;22:103-6.

51 Fitzgerald GK, Piva SR, Gil AB, et al. Agility and perturbation training techniques in exercise therapy for reducing pain and improving function in people with knee osteoarthritis: a randomized clinical trial. Phys Ther 2011;91:452-69.

52 Fransen M, Crosbie J, Edmonds J, et al. Physical therapy is effective for patients with osteoarthritis of the knee: a randomized controlled clinical trial. J Rheumatol 2001:28:156-64.

53 Gaudreault N, Mezghani N, Turcot K, et al. Effects of physiotherapy treatment on knee osteoarthritis gait data using principal component analysis. Clinical Biomechanics 2011;26:284-91.

54 Gomes WF, Lacerda ACR, Mendonça VA, et al. Effect of aerobic training on plasma cytokines and soluble receptors in elderly women with knee osteoarthritis, in response to acute exercise. Clin Rheumato/ 2012;31:759-66.

55 Gomes WF, Lacerda ACR, Mendonça VA, et al. Effect of exercise on the plasma BDNF levels in elderly women with knee osteoarthritis. Rheumatol Int 2014:34:841-6.

56 Gomes WF, Lacerda ACR, Brito-Melo GEA, et al. Aerobic training modulates T cell activation in elderly women with knee osteoarthritis. Braz J Med Biol Res 2016;49:1-9

57 Hunt MA, Keefe FJ, Bryant C, et al. A physiotherapist-delivered, combined exercise and pain coping skills training intervention for individuals with knee osteoarthritis: a pilot study. Knee 2013;20:106-12.

58 Kabiri S, Halabchi F, Angoorani H, et al. Comparison of three modes of aerobic exercise combined with resistance training on the pain and function of patients with knee osteoarthritis: a randomized controlled trial. Physical Therapy in Sport 2018:32:22-8

59 Kappetijn 0, van Trijffel E, Lucas C. Efficacy of passive extension mobilization in addition to exercise in the osteoarthritic knee: an observational parallel-group study. Knee 2014:21:703-9.

60 Keefe FJ, Blumenthal J, Baucom D, et al. Effects of spouse-assisted coping skills training and exercise training in patients with osteoarthritic knee pain: a randomized controlled study. Pain 2004;110:539-49.

61 Koli J, Multanen J, Kujala UM, et al. Effects of exercise on Patellar cartilage in women with mild knee osteoarthritis. Med Sci Sports Exerc 2015;47:1767-74.

62 Kovar PA, Allegrante JP, MacKenzie CR, et al. Supervised fitness walking in patients with osteoarthritis of the knee: a randomized, controlled trial. Ann Intern Med 1992;116:529-34.

63 Kuptniratsaikul V, Kittichaikarn C, Suntornpiyapan P, et al. Is four-week underwater treadmill exercise regimen compared to home exercise efficacious for pain relief and functional improvement in obese patients with knee osteoarthritis? A randomized controlled trial. Clin Rehabil 2019;33:85-93. 
64 Larose J, King J, Brosseau L, et al. The effect of walking on cardiorespiratory fitness in adults with knee osteoarthritis. Appl Physiol Nutr Metab 2013:38:886-91.

65 Lau M, Lam J, Siu E, et al. Physiotherapist-designed aquatic exercise programme for community-dwelling elders with osteoarthritis of the knee: a Hong Kong pilot study. Hong Kong Med J 2014;20:16-23.

66 Lim J-Y, Tchai E, Jang S-N. Effectiveness of aquatic exercise for obese patients with knee osteoarthritis: a randomized controlled trial. $P M \& R$ 2010;2:723-31.

67 Lund $\mathrm{H}$, Weile $\mathrm{U}$, Christensen $\mathrm{R}$, et al. A randomized controlled trial of aquatic and land-based exercise in patients with knee osteoarthritis. J Rehabil Med 2008:40:137-44.

68 Mangione KK, McCully K, Gloviak A, et al. The effects of high-intensity and lowintensity cycle ergometry in older adults with knee osteoarthritis. J Gerontol A Biol Sci Med Sci 1999;54:M184-90.

69 Messier SP, Loeser RF, Mitchell MN, et al. Exercise and weight loss in obese older adults with knee osteoarthritis: a preliminary study. J Am Geriatr Soc 2000;48:1062-72.

70 Messier SP, Loeser RF, Miller GD, et al. Exercise and dietary weight loss in overweight and obese older adults with knee osteoarthritis: the arthritis, diet, and activity promotion trial. Arthritis Rheum 2004;50:1501-10.

71 Messier SP, Mihalko SL, Legault C, et al. Effects of intensive diet and exercise on knee joint loads, inflammation, and clinical outcomes among overweight and obese adults with knee osteoarthritis. JAMA 2013;310:1263-73.

72 Nicklas BJ, Ambrosius W, Messier SP, et al. Diet-Induced weight loss, exercise, and chronic inflammation in older, obese adults: a randomized controlled clinical trial. Am J Clin Nutr 2004;79:544-51.

73 Péloquin L, Bravo G, Gauthier $\mathrm{P}$, et al. Effects of a cross-training exercise program in persons with osteoarthritis of the knee a randomized controlled trial. J Clin Rheumatol 1999:5:126-36.

74 Rosa UH, Velásquez Tlapanco J, Lara Maya C, et al. Comparison of the effectiveness of isokinetic vs isometric therapeutic exercise in patients with osteoarthritis of knee. Reumatol Clin 2012:8:10-14.

75 Rosemffet MG, Schneeberger EE, Citera G, et al. Effects of functional electrostimulation on pain, muscular strength, and functional capacity in patients with osteoarthritis of the knee. J Clin Rheumatol 2004;10:246-9.

76 Salacinski AJ, Krohn K, Lewis SF, et al. The effects of group cycling on gait and pain-related disability in individuals with mild-to-moderate knee osteoarthritis: a randomized controlled trial. J Orthop Sports Phys Ther 2012:42:985-95.

77 Samut G, Dinçer F, Özdemir O. The effect of isokinetic and aerobic exercises on serum interleukin-6 and tumor necrosis factor alpha levels, pain, and functional activity in patients with knee osteoarthritis. Mod Rheumato/ 2015;25:919-24.

78 Singh J, Singh P, Sohal MS, et al. Effect of exercise rehabilitation programme on clinical health status of osteoarthritis knee patients. Indian J Physiother Occup Ther 2011:5:191-8.

79 Somers TJ, Blumenthal JA, Guilak F, et al. Pain coping skills training and lifestyle behavioral weight management in patients with knee osteoarthritis: a randomized controlled study. Pain 2012;153:1199-209.

80 Thorstensson CA, Roos EM, Petersson IF, et al. Six-week high-intensity exercise program for middle-aged patients with knee osteoarthritis: a randomized controlled trial [ISRCTN20244858]. BMC Musculoskelet Disord 2005;6:1-10.

81 Wallis JA, Webster KE, Levinger $P$, et al. A walking program for people with severe knee osteoarthritis did not reduce pain but may have benefits for cardiovascular health: a phase II randomised controlled trial. Osteoarthritis and Cartilage 2017:25:1969-79.

82 Wang T-J, Lee S-C, Liang S-Y, et al. Comparing the efficacy of aquatic exercises and land-based exercises for patients with knee osteoarthritis. J Clin Nurs 2011;20:2609-22.

83 Yennan P, Suputtitada A, Yuktanandana P. Effects of aquatic exercise and land based exercise on postural sway in elderly with knee osteoarthritis. Asian Biomed 2010:4:739-45

84 ACSM. Exercising with osteoarthritis. Available: https://www.exerciseismedicine.org/ assets/page_documents/EIM Rx series_Exercising with Osteoarthritis.pdf.

85 Bricca A, Juhl CB, Steultjens $M$, et al. Impact of exercise on articular cartilage in people at risk of, or with established, knee osteoarthritis: a systematic review of randomised controlled trials. Br J Sports Med 2019:53:940-7.

86 Collins NJ, Hart HF, Mills KAG. Osteoarthritis year in review 2018: rehabilitation and outcomes. Osteoarthritis and Cartilage 2019:27:378-91.

87 Boudou P, De Kerviler E, Vexiau P, et al. Effects of a single bout of exercise and exercise training on steroid levels in middle-aged type 2 diabetic men: relationship to abdominal adipose tissue distribution and metabolic status. Diabetes Metab 2000:26:450-7.

88 Wewege MA, Thom JM, Rye K-A, et al. Aerobic, resistance or combined training: a systematic review and meta-analysis of exercise to reduce cardiovascular risk in adults with metabolic syndrome. Atherosclerosis 2018;274:162-71.

89 Huang G, Gibson CA, Tran ZV, et al. Controlled endurance exercise training and V02max changes in older adults: a meta-analysis. Prev Cardio/ 2005:8:217-25.

90 Huang G, Shi X, Gibson CA, et al. Controlled aerobic exercise training reduces resting blood pressure in sedentary older adults. Blood Press 2013;22:386-94.

91 Kohut ML, McCann DA, Russell DW, et al. Aerobic exercise, but not flexibility/ resistance exercise, reduces serum IL-18, CRP, and IL- 6 independent of $\beta$-blockers, BMI, and psychosocial factors in older adults. Brain Behav Immun 2006;20:201-9.

92 Beavers KM, Brinkley TE, Nicklas BJ. Effect of exercise training on chronic inflammation. Clinica Chimica Acta 2010;411:785-93.

93 Hall AJ, Stubbs B, Mamas MA, et al. Association between osteoarthritis and cardiovascular disease: systematic review and meta-analysis. Eur J Prev Cardiol 2016;23:938-46.

94 Hsu P-S, Lin H-H, Li C-R, et al. Increased risk of stroke in patients with osteoarthritis: a population-based cohort study. Osteoarthritis and Cartilage 2017;25:1026-31.

95 Calvet J, Orellana C, Larrosa M, et al. High prevalence of cardiovascular comorbidities in patients with symptomatic knee or hand osteoarthritis. Scand J Rheumatol 2016:45:41-4.

96 Brosseau L, Taki J, Desjardins B, et al. The Ottawa panel clinical practice guidelines for the management of knee osteoarthritis. Part three: aerobic exercise programs. Clin Rehabil 2017:31:612-24.

97 Roddy Eet al. Aerobic walking or strengthening exercise for osteoarthritis of the knee? A systematic review. Ann Rheum Dis 2005:64:544-8.

98 Fransen M, McConnell S. Land-based exercise for osteoarthritis of the knee: a metaanalysis of randomized controlled trials. J Rheumatol 2009:36:1109-17.

99 Dunlop DD, Song J, Lee J, et al. Physical activity minimum threshold predicting improved function in adults with Lower-Extremity symptoms. Arthritis Care Res 2017;69:475-83

100 Cuijpers P, Weitz E, Cristea IA, et al. Pre-Post effect sizes should be avoided in metaanalyses. Epidemiol Psychiatr Sci 2017;26:364-8. 